\title{
Determinación del riesgo de caries en niños menores de 5 años en escuelas del sector norte de Quito, Ecuador
}

\section{Determination of the caries risk in children under 5 years of age in schools of the northern of Quito, Ecuador}

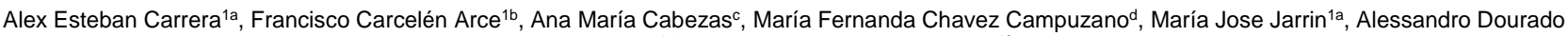
Loguercio ${ }^{1,3 e}$, Ana del Carmen Armas Vega ${ }^{1 \mathrm{f}}$

\section{RESUMEN}

Objetivo: Estimar el riesgo de caries de los niños menores de 5 años de la zona urbana del sector norte de Quito, Ecuador. Materiales y métodos: La muestra quedó conformada por 102 pares; niño y padres y/o representantes, quienes previa autorización mediante consentimiento informado firmado por los padres y/o representante y asentimiento informado firmado por niño, fueron solicitados a llenar la encuesta Caries Management by Risk Assessment (CAMBRA) para determinar el riesgo de presentar caries en sus hijos. También fue hecho en examen clínico para avaliar la presencia de caries y placa Los datos fueron recopilados a una tabla de Excel y fueron sometidos a análisis estadísticos descriptivos. Resultados: $60,8 \%$ de los menores participantes pertenecieron al género femenino. El cuestionario CAMBRA permitió obtener la valoración de riesgo alto. Mientras, el examen clínico ejecutado permitió determinar que el índice de caries fue de 4,62 dientes temporales afectados con la presencia de placa en un $86 \%$ de la población evaluada. Conclusiones: Según la encuesta CAMBRA aplicada, el riesgo a presentar caries es alto y guarda gran relación con los índices de caries y placa dental detectados en la misma población.

Palabras clave: caries dental, susceptibilidad a caries dentales, primerainfancia, Cuidado del Niño. (Fuente: DeCS BIREME)

\section{ABSTRACT}

Objective: Estimated the caries risk of children under 5 years of age in the urban area of the northern sector in Quito, Ecuador. Materials and methods: The sample consisted by 102 pairs; child and parents and / or representatives, who have been accepted a prior informed consent, they were subjected to the management of caries by risk assessment survey (CAMBRA) to determine the risk of presenting caries in their children. We were also done a clinical examination to assess the presence of caries and plaque. The data was compiled in an Excel table and were analyzed in SPSS program. Results: $60.8 \%$ of the minor participants belonging to the female gender. CAMBRA survey has a high-risk measure. Meanwhile, the clinical examination determined that the temporal teeth caries index was 4.62 with the presence of a plaque in $86 \%$ of the evaluated population. Conclusions: According to the survey, CAMBRA applied, the risk to the presentation of caries is high and is related to the rates of tooth decay and dental plaque detected in the same population.

Keywords: dental caries, risk managment, dental caries susceptibility, Child Care. (Source: MeSH NLM)

\footnotetext{
1 Universidad Tecnológica Equinoccial

2 Universidad San Francisco de Quito

${ }^{3}$ Universidad Estadual de Ponta Grossa

a Odontólogo

b Estudiante de Odontología

${ }^{c}$ Especialista en Odontopediatría

dEspecialista en Rehabilitación Oral

e PhD en Materiales Dentales

${ }^{f} \mathrm{PhD}$ en Operatoria Dental
}

Correspondencia

Alex Esteban Carrera Robalino

Correo Electrónico: alexcr199415@gmail.com

Este es un artículo Open Access distribuido bajo la licencia Creative Commons Atribución-NoComercialCompartir Igual 4.0

무(1)(2)

Citar como: Carrera A, Carcelén F, Cabezas A, Chávez M, Jarrin M, Dourado A, Armas A. Determinación del riesgo de caries en niños menores de 5 años en escuelas del sector norte de Quito, Ecuador. KIRU. 2019; abril-jun; 16(2): 69-74. https://doi.org/10.24265/kiru.2019.v16n2.03 


\section{INTRODUCCIÓN}

La caries dental se considera un proceso dinámico crónico, infeccioso, transmisible y multifactorial (1) caracterizado por la destrucción de los tejidos duros del diente ${ }^{(2)}$. Esta patología ocurre sin distinción género, raza, ubicación demográfica o estado sociocultural y económico e inclusive edad, constituyéndose en la enfermedad más común a nivel bucal ${ }^{(3,4)}$.

Su presencia guarda estrecha relación con el metabolismo de los diferentes microorganismos presentes en la placa dental, ${ }^{(3,5)}$, con una notable influencia de la dieta en su presencia ${ }^{(6)}$ asociada a su consistencia, textura y adhesión (6), actuando como factores predisponentes, obligando establecer protocolos de prevención y cuidado de los dientes principalmente en la primera infancia ${ }^{(7)}$.

La asociación de la caries y el bajo nivel socioeconómico guarda relación al limitado acceso a la asistencia médica (8), como fuera demostrado en estudios previos ejecutados en países vecinos como Colombia ${ }^{(9)}$. Por otro lado, poco se sabe sobre o riesgo de estos pacientes en desarrollar caries dentarias. El riesgo individual de cada paciente a la patología por medio de indicadores de la enfermedad y factores biológicos de riesgo que sirven de base para estructurar tratamientos posteriores ${ }^{(7)}$ así como los factores protectores de cada paciente buscando reforzar conductas de prevención y cuidado para la salud bucal para implementarlas en los niños ${ }^{(10)}$.

Recientemente, se ha trabajado el manejo de la caries mediante la valoración de riesgo CAMBRA (Caries Management by Risk Assessment). Este es considerado una buena herramienta aplicable a la práctica clínica diaria para identificar la susceptibilidad del paciente a la caries dental (11). Sin embargo, no existen estudios aplicando el CAMBRA y asociando con los índices de caries dental y placa en países de Latinoamérica. Así mediante este estudio se pretende estimar el riesgo de presentar caries que refieren los niños menores de 5 años y su asociación con la presencia de caries y placa en los mismos.

\section{MATERIALES Y MÉTODOS}

Se realizó un estudio transversal descriptivo, en una población establecida por conveniencia de 254 familias que acuden a recibir atención en los centros de salud Zambiza, Cocotog, Nayón pertenecientes al Ministerio de Salud Pública del Ecuador ubicados en la zona urbano marginal del norte de Quito, Ecuador conformado por: Cochapamba, Nayón, Zambiza, Cocotog, Tanda y La Pulida entre Julio y Septiembre del 2017. El proyecto formó parte de un levantamiento de necesidades de atención realizado en convenio entre la Universidad Tecnológica Equinoccial y el Ministerio de Salud Pública del Ecuador.
De las 254 familias que constituyeron la población, deberían cumplir los criterios de inclusión: contar con un miembro en la familia menor de 5 años de edad, tener la capacidad de contar con el responsable o representante de la familia en condiciones y con predisposición para entregar información, recibir atención médica en uno de los centros de atención pública involucrados en el estudio, el contar con la firma del padre y/o representante en el documento consentimiento informado, así como la firma del menor en la carta de asentimiento informado y el contar con la presencia del menor en el momento de realizar la encuesta. Fueran excluidos aquellos niños con enfermedades sistémicas que comprometieran su salud bucal o general o que se encontraban bajo tratamiento médico. De esta manera, la muestra quedó conformada por 102 pares, niño y padres de familia y/o representantes.

Los padres de familia tras recibir explicación del estudio y sus implicaciones fueron solicitados a llenar el cuestionario CAMBRA como instrumento para la evaluación de riesgo de caries (Tabla 1). El cuestionario fue obtenido de la página web de la Sociedad Española de Odontopediatria y fue aplicado por un investigador previamente entrenado y calibrado en recolectar información de este cuestionario, con la supervisión de un docente investigador a cargo del proyecto previamente entrenado y calibrado. La identidad del padre y/o participante fue preservada empleándose códigos para identificar cada una de las fichas.

El cuestionario CAMBRA modificado y específico para edades de 0 a 5 años, se presentó en idioma español y fue presentada cada pregunta por el entrevistador. El cuestionario consta de 14 preguntas dicotómicas (Si o No) divididas en 4 grupos, seguida de la valoración según el caso. Frente a ciertas preguntas que exigían la evaluación clínica del menor, bajo la presencia y autorización del padre, la boca del menor fue examinada bajo los parámetros de bioseguridad empleándose fronto luz, espejo bucal y sonda OMS. Esta parte fue realizada por parte del evaluador a cargo. Quien de forma complementaria registró en la ficha la presencia de lesiones cariosas, restauraciones dentales o ausencias dentales según el índice ceod y obteniendo un valor promedio considerando el número de dientes pertenecientes a la dentición decidua.

De la misma manera fue verificada la presencia de placa considerando el índice de Loe y Stilness que considera el examen de seis dientes temporales o definitivos y asocia con códigos determinados para cada diente donde 0 es considerado con ausencia de placa, 1 a simple vista, no hay placa pero se observa al paso de un instrumental, 2 existe placa a simple vista y 3 existe blanca a simple vista incluso en proximal. 
Tabla 1. Cuestionario CAMBRA modificado para edades de 0 a 5 años

Nombre del alumno:

Edad:

Grado:

Fecha de encuesta:

Pregunta

¿Ha tenido la madre o cuidador principal caries en el último año?

¿Se le ha realizado al niño alguna obturación (calza) recientemente?

¿Tiene la madre o cuidador principal nivel socio económico o cultural bajo?

¿Tiene problemas de desarrollo?

¿No realiza visitas al dentista de forma periódica?

¿Toma snacks o bebidas azucaradas entre horas más de tres veces diarias?

¿Tiene reducción de la producción de saliva por medicación u otras causas?

¿Bebe habitualmente en botella o biberón bebidas que no sean agua?

¿Duerme con biberón o toma pecho a demanda mientras duerme?

La madre o cuidador no ha tenido caries en los últimos tres años

Realiza revisiones y controles periódicos con un dentista

Vive en un área con agua fluorada o deshace en boca comprimidos fluorados

Se cepilla con pasta fluorada diariamente (tamaño lenteja o guisante)

La madre utiliza chicles o pastillas con xilitol 2-4 veces al día

PUNTUACION

PUNTUACION TOTAL:

NIVEL DE RIESGO:

Los datos recolectados de la encuesta fueron tabulados siguiendo las valoraciones que la propia encuesta señala considerando el puntaje y el riesgo presentar caries dental, de acuerdo con la recomendación del CAMBRA, o sea, $\leq 5$ como Bajo riesgo y $\geq 6$ como de Alto riesgo. Los valores determinados de riesgo fueron cruzados con el género, escolaridad o no y edad del menor participante y el centro de salud al que frecuentaba de entre los cinco considerados dentro del estudio.

Posterior a la evaluación del riesgo se realizó charlas de promoción y prevención de caries en las instituciones involucradas para colaborar a que se reduzcan aquellos factores de riesgo y se potencien los factores de prevención para la caries. Los resultados obtenidos recolectados en fichas en el programa Excel 2010 fueron tabulados y procesados mediante el programa Statistics, empleándose la correlación lineal de Pearson para la obtención de la significancia estadística (valor de p).

\section{RESULTADOS}

De los 102 padres y sus respectivos hijos fueron encuestados, según los datos recopilados el $60,8 \%$ de los menores pertenecieron al género femenino. La información recolectada de las preguntas que constituyen el cuestionario CAMBRA permitió obtener la valoración de riesgo, observando que el $74,7 \%$ de la población analizada presenta un alto riesgo a caries.

Con respecto al examen clínico ejecutado para valorar la presencia de caries, mediante el índice ceod, permitió determinar que el índice de caries fue de 4,62 en promedio en los dientes temporales afectados. Observándose que el indicador diente cariado fue el más alto, seguido por el indicador diente obturado. La mayoría de las piezas extraídas fueron debido a caries $(95 \%)$ y el restante $5 \%$ se extrajeron debido a traumatismos en la zona orofacial. Con respecto a la placa la presencia de placa constatada tras el examen clínico revelo un índice de placa de placa 1 según el índice de Loe y Stilness en un $86 \%$ de la población evaluada.

Se encontró una significancia estadísticamente significativa entre el riesgo alto de caries $(74,7 \%)$ y el alto índice de caries presentado $(p<0,01)$. También fue encontrada una relación estadísticamente significativa entre la presencia de caries y el alto índice ceod $(p<0,0001)$, teniendo una mayor presencia de dientes cariados sin tratamiento previo. 


\section{DISCUSIÓN}

Los resultados del estudio muestran una tendencia en los participantes a presentar alto riesgo de caries, coincidiendo con el examen clínico ejecutado que revelo valores de caries y presencia de placa altos. Además, esta relación directa entre el alto riesgo de caries y el alto índice de caries encontrado, nos indica que el alto riesgo a caries aumenta la probabilidad de presentar caries.

Si bien en las zonas de residencia de los encuestados, las entidades de gobierno han desarrollado actividades de promoción y prevención de salud, que resultan importantes cuando prevención y control de riesgo de la enfermedad se trata ${ }^{(7,12,13)}$, en la evaluación clínica de los menores fue observada la elevada presencia de dientes en estado de deterioro que fue considerado clínicamente como dientes cariado y restaurados. Esto evidencia el trabajo que se viene ejecutando en los centros de salud intervenidos; pero que están resultando insuficientes, según lo apreciado en la evaluación clínica.

Es evidente que al realizar cambios en los factores de riesgo dieta o higiene oral en la madre, esta actuara como elemento de difusión y replica hacia el niño, lo que constituye la mejor herramienta de control de la enfermedad (14,15). Aparentemente este envolvimiento de la familia en el proceso salud de enfermedad es el punto que requiere ser reforzado en esta población, pues solo así se explica el por qué la encuesta de riesgos muestra un resultado negativo interpretado en un riesgo alto de caries y el análisis de índices de caries y placa realizado con las limitaciones que un examen clínico ejecutado en campo ofrece muestran elevados valores de estos dos indicadores.

La valoración de riesgo de caries mediante la encuesta CAMBRA, es considerada una buena herramienta aplicable a la práctica clínica dental ${ }^{(16)}$ para conocer la susceptibilidad del individuo de presentar caries $(7,11)$, permitiendo ser aplicada individualmente y proporciona un pronóstico de la patología, altamente confiable. Sin embargo, requiere la colaboración de los padres sobre todo cuando la muestra está constituida por niños menores de 5 años como en este caso. Por lo tanto, una de las limitaciones que el estudio presenta es la ausencia de información sobre el sujeto padre y/o representante que proporciona la información, pues la entrega de información por parte de esta persona constituye la clave principal del éxito y de los resultados a obtenerse.

Estudios similares donde la encuesta CAMBRA ha sido empleada, conjugan la evaluación clínica del estado de salud bucal $(7,17)$, lo que motivo la ejecución de este estudio. Estos, en general, demuestran resultados similares al da presente investigación $(7,13,17)$. Sin embargo, es fundamental contar con un personal evaluador capacitado en presentar esas preguntas a los padres y con conocimiento para la identificación de ciertos aspectos básicos cuando se trata de considerar preguntas sobre la morfología de las superficies dentales $(18,19)$, presentando algunos problemas en la subestimación del riesgo, sobre todo en la evaluación clínica ${ }^{(20)}$.

Por otro lado, se hace imprescindible contar con la colaboración de quien proporciona la información en cuanto a la veracidad de esta, observándose que la percepción de un aspecto estará determinada por diferentes elementos, siendo el nivel de educación, motivación e incluso género de la persona (3), fundamentales en el momento de obtener una información confiable, considerándose como una limitante del estudio.

Otro aspecto a considerar es el hecho de que la encuesta CAMBRA cuenta con un alto nivel de sensibilidad, pero baja especificidad ${ }^{(11,21,22)}$, por lo que puede proporcionar alto nivel de falsos positivos en la ejecución del estudio. Las predicciones en cuanto a riesgo, si bien son básicas, exigen ser complementadas con otras valoraciones, y posiblemente conocer el estado de salud de los padres y/o representantes, se constituyan en herramienta previsora ${ }^{(23)}$. Esto es además importante cuando se trata de que en menores de 5 años son estos los responsables directos de la ejecución de técnicas de cepillado y por tanto de la presencia o no de caries dental ${ }^{(7,24)}$.

Una de las grandes limitantes del estudio es la falta de realización del estudio de la cantidad de Streptocuccus spp. en boca de los participantes por falta de recursos, siendo una variable a tomar en cuenta en estudios posteriores a realizar.

Estudios siguiendo la misma metodología requieren ser ejecutados, considerando otros indicadores de salud bucal, como ICDAS y su valoración por superficie y gravedad.

Las autoridades de salud dentro de las políticas del gobierno ecuatoriano realizan diferentes campañas, sin embargo, la valoración de la efectividad solo puede ser determinada con estudios epidemiológicos. si bien la muestra que formó parte del estudio fue relativamente pequeña no difiere a estudios con similar metodología $(13,25)$ y nos abre el camino para continuar con investigación que ayuden a nuestra comunidad, para plantearnos estrategias y metas que requieren ser cumplidas sobre todo en lo que respecta a la salud bucal de menores de 5 años.

Se concluye, según la encuesta CAMBRA aplicada, que el riesgo a presentar caries en la muestra evaluada es alto y guarda gran relación con los índices de caries y placa dental detectada.

Contribuciones de autoría: AEC, FCA, AMC, MFCC, MJJ, ADL, AAV diseñaron el estudio, recopilaron y analizaron los datos. Los autores redactaron y aprobaron la versión final del artículo. 
Fuente de financiamiento: El estudio fue financiado por los autores.

Conflicto de intereses: Los autores declararon no tener conflictos de interés.

\section{REFERENCIAS}

1. Astorga, B., Barraza, C., Casals, J. M., Cisterna, M. J., Mena, D., Morales, F., ... \& Moncada, G. (2015). Avances en el estudio de la diversidad bacteriana oral asociada a caries dental mediante el estudio genómico. Int. J. Odontostomat. 9(3), 349-356.

2 Martínez JC, Alegría J. Polimorfismos genéticos y su influencia en la aparición y evolución de infecciones dentales. REDICINAySAuniversidad de Guanajuato. [Internet] 2017 [citado: 31/01/2018]; 6(1): 15-18. Disponible en: http://www.ugto.mx/redicinaysa/images/publicac iones/2017/Enero-Febrero_2017redicinaysa.pdf

3. Young DA, Featherstone JD. Caries management by risk assessment. Community Dent Oral Epidemiol. 2013 Feb; 41(1).e53-63.

4. Cárdenas C, Perona G. Factores de riesgos asociados a la prevalencia de caries de aparición temprana en niños de 1 a 3 años en una población peruana. Odontolol Pediatr. 2013; 12(2). 110-118.

5. Mira A, Soro A. De la etiología a la clínica en la caries dental. Gaceta dental. 2015; (266):102112.

6. Mayor Hernández Félix, Pérez Quiñones José Alberto, Cid Rodríguez María del Carmen, Martínez Brito Isabel, Martínez Abreu Judit, Moure Ibarra Miriam D. La caries dental y su interrelación con algunos factores sociales. Rev. Med. Electrón. [Internet]. 2014 Jun [citado: 31/01/2018]; 36( 3 ): 339-349. Disponible en: http://scielo.sld.cu/scielo.php?script=sci_arttext \&pid=S1684-18242014000300010\&lng=es

7. López A, Mateos M, Bratos E, Garcillán M. Diagnóstico del riesgo de caries en los pacientes infantiles de la Universidad Complutense de Madrid. Sociedad española de odontopediatria (SEOP). Odontología Pediátrica (Madrid). 2018; (24) 3:183-193.

8. Pautasso R, Fonseca C, Córdoba P. La caries dental y factores relacionados en una poblaciónde adultos de la ciudad de la Rioja, Argentina. Acta Odontol. Venez. 2014; 52 (2)

9. Zuñiga $\mathrm{E}$, Porras J. Comportamiento epidemiológico de la caries dental en Colombia. Univ. Odontol. 2013 Ene-Jun; 32(68): 117-124.
10. Hallett KB. The application of caries risk assessment in minimum intervention dentistry. Aust Dent J. 2013;58:26-34.

11. Doméjean $S$, Léger $S$, Rechmann $P$, White JM, Featherstone JDB. How do dental students determine patients' caries risk level using the caries management by risk assessment (CAMBRA) system. J Dent Educ 2015; 79(3):27885.

12 Ramos-Gomez, MW Ng. Into the future: keeping healthy teeth caries free: paediatric CAMBRA protocols. J Calif Dent Assoc. 2011; 39 (10): 723733.

13. Featherstone, J., Chaffee, B. The Evidence for Caries Management by Risk Assessment (CAMBRA®). Adv Dent Res. 2018; 29(1): 9-14.

14. Meyer, F. and Enax, J. Early Childhood Caries: Epidemiology, Aetiology, and Prevention. Int. J. Dent., 2018: 1-7.

15. Halawany $\mathrm{H}, \mathrm{Al}$ Badr A, AI Sadhan S, Al Balkhi M, Al-Maflehi N, Abraham N, Jacob V, Al Sherif G. Effectiveness of oral health education intervention among female primary school children in Riyadh, Saudi Arabia. Saudi Dent J. 2018 Jul; 30(3): 190196.

16. Sudhir KM, Kanupuru KK, Fareed N, Mahesh $\mathrm{P}$, Vandana K, Chaitra NT. CAMBRA as a Tool for Caries Risk Prediction Among 12- to 13-year-old Institutionalised Children - A Longitudinal Followup Study. Oral Health Prev Dent. 2016;14(4):35562. doi: 10.3290/j.ohpd.a35621.

17. Gauba K, Goyal A, Mittal N. A CAMBRA Model For High Caries Risk Indian Children: A Pragmatic Comprehensive Tailored Intervention. Int. J. Clin. Pediatr. Dent.2016; 40(1), pp.36-43.

18. Goolsby SP, Young DA, Chiang HK, Carrico CK, Jackson LV, Rechmann P. The Effects of Faculty Calibration on Caries Risk Assessment and Quality Assurance. J Dent Educ. 2016 Nov;80(11):1294-1300.

19. Young DA, Alvear Fa B, Rogers N, Rechmann P. The Effect of Calibration on Caries Risk Assessment Performance by Students and Clinical Faculty. J Dent Educ. 2017 Jun;81(6):667-674.

20. Teich ST, Demko C, Al-Rawi W, Gutberg T. Assessment of implementation of a CAMBRAbased program in a dental school environment. $\mathrm{J}$ Dent Educ. 2013 Apr;77(4):438-47.

21. Gao XL, Lo EC, Chu CH, Hsu SC. Caries risk assessment programmes for Hong Kong children. Hong Kong Med J. 2015 Dec;21 Suppl 6:42-6. PubMed PMID: 26645883. 
22 Rechmann P, Featherstone JD. Quality assurance study of caries risk assessment performance by clinical faculty members in aschool of dentistry. J Dent Educ. 2014 Sep;78(9):1331-8.

23. Cheng J, Chaffee BW, Cheng NF, Gansky SA, Featherstone JD. Understanding treatment effect mechanisms of the CAMBRA randomized trial in reducing caries increment. J Dent Res. 2015 Jan;94(1):44-51.
24. Villaizán C., Aguilar M. Estudio de la prevalencia de caries y su relación con factores de higiene oral y hábitos cariogénicos en escolares. Revista Latinoamericana de Ortodoncia y Odontopediatría "Ortodoncia.ws edición electrónica Mayo 2012. Disponible en: www.ortodoncia.ws. Consultada, 26/06/2018

25. Graciano M., Correa Y., Martinez C., Burgos A., Ceballos J., Sanchez L. (2012).Streptococcus mutans y caries dental en america latina. Revisión sistematica de la literatura. Rev. Nacional de odontología. Colombia. 8(14):32-45. 